\section{JONES MATRIX POLARIMETRY FOR HIGH POWER LASER OPTICAL COMPONENTS}

ONDREJ SLEZAK AND ANTONIO LUCIANETTI

HiLASE Centre, Institute of Physics, Czech Academy of Sciences, Dolni Brezany, Czech Republic

DOI : 10.17973/MMSJ.2019_12_2019113

e-mail : ondrej.slezak@hilase.cz

The Jones matrix based polarimetric method suitable for the spatially resolved analysis of the thermally induced birefringence and dichroism in complex high-power laser optical systems is proposed. The measurement allows the characterization of the complete local Jones matrix of the optical components, or their sets. The method of extraction of the particular contributions of the linear and circular birefringence and dichroism is also suggested in order to recognize the phenomena and effects contributing to the total induced polarization state distortions. The whole procedure is demonstrated on the simulated experiment which analyses a very general inhomogeneous polarization distorting piece of material.

\section{KEYWORDS:}

Polarimetry, ellipsometry, Birefringence, Dichroism, depolarization

\section{INTRODUCTION}

The continual development of high average power diode pumped solid state lasers in the recent years [Mason 2017, Reagan 2018, Zapata 2015, Novak 2015] led to the urgent need of the analysis and the measurement of the thermal effects induced in the laser amplifiers and other optical components. Numerous methods of calculations of the thermally induced wavefront distortions and thermally induced birefringence starting from analytical methods for highly symmetrical geometries [Koechner 1970], and gradually improving to the more sophisticated numerical approaches [Slezak 2013, Graupeter 2015, Slezak 2015, Ribes-Pleguezuelo 2017], were proposed in the past. The necessity of the experimental verification of these calculations also led to the development of the measurement methods for temperature distributions [Körner 2018, Chi 2018] and depolarization of the laser beam caused by the thermal stress induced birefringence [Lumer 2010, Yasuhara 2013]. The later led also to the development of several methods of the compensation of thermal stress induced birefringence mostly by usage of reciprocal rotator placed between two identical thermally loaded optical components [Kagan 2003, Snetkov 2011]. Nevertheless, the measurement of the depolarization (defined as the ratio of the power transmitted through the optical system placed between the crossed polarizers and the input power) cannot provide the information about the origin of the polarization distorting effects which contributed to the overall polarization distortion. Another disadvantage of such simple characterization of the induced birefringence is the impossibility to subtract some parts of the system, while in complex laser system it is usually impossible to do the measurement for each isolated component and just the measurement of a relatively numerous sets of components is possible, to obtain an isolated contribution of another part. The later comes from the fact that the depolarization is not an additive quantity. All these disadvantages could be overcame by the usage of some polarimetric method which characterizes the full Jones or Mueller matrix of the optical system [Goldstein 1992, Savenkov 2007]. An overview of the Mueller matrix polarimetry can be found in the literature [Azzam 2016]. However, it is exclusively a fully polarized light which is propagating through the laser system and therefore the Jones matrix polarimetry could be used instead of Mueller matrix polarimetry. In this paper, we will design and analyze one potential method of the induced polarization effects analysis by Jones matrix polarimetry.

\section{POLARIMETRIC MEASUREMENT}

\subsection{Polarimeter description}

In the following paper one has to deal with the matrices of several dimensions. To distinguish uniquely among those types, let us use following notation:

\begin{tabular}{cc|ll}
\hline Matrix & Symbol & Range & Indices \\
\hline $2 \times 2$ & $\mathbf{M}$ & $1 . .2$ & $\mathrm{a}, \mathrm{b}, \mathrm{c}, \mathrm{d}$ \\
$4 \times 4$ & $\bar{M}$ & $1 . .4$ & $\mathrm{i}, \mathrm{j}, \mathrm{k}, \ldots$ \\
$4 \times 6$ & $\widehat{M}$ & $1 . .6$ & $\mu, \mathrm{v}, \ldots$ \\
\hline
\end{tabular}

Table 1. Matrix types notation

The experimental setup used for the measurement of the set of transmitted intensities is shown in the Fig. 1.

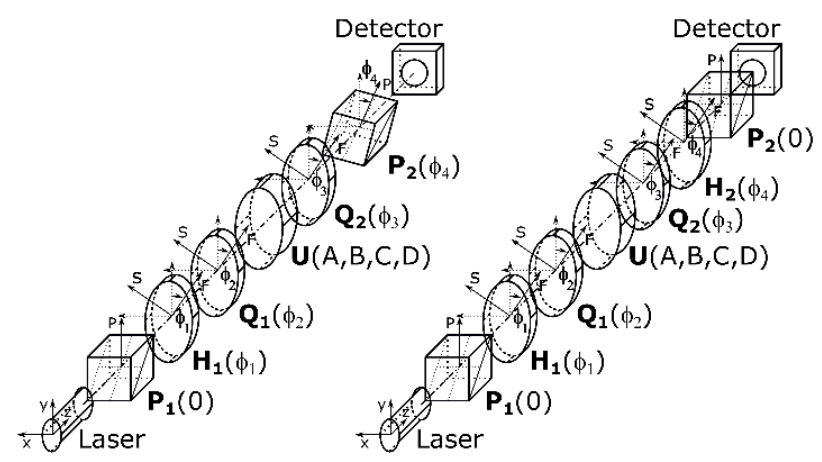

a)

b)

Figure 1. Schematic of experimental setup for Jones polarimetry. $\mathrm{P}$ - fixed polarizer, $\mathrm{H}$ - Half-wave plate, $\mathrm{Q}$ - Quarter-wave plate, $\mathrm{U}$ - sample. $\mathrm{F}$ and $\mathrm{S}$ denotes the fast and slow axes of the waveplates.

It should be noted, that there are two almost equivalent schemes shown here. Scheme a) contains a rotating analyzer whereas the scheme $b$ ) has the rotating analyzer replaced with the combination of rotating half-wave plate and fixed angle analyzer for the cases, when it is inconvenient to rotate the polarizer. All the equations in the following text will be derived for the case a). The transformation into the case b) is a simple substitution of $\phi_{4} / 2$ instead of $\phi_{4}$.

$$
\phi_{4}^{b)}=\frac{\phi_{4}^{a)}}{2} \text {. }
$$

The general Jones matrix representing the sample under investigation which can possess linear and circular birefringence and dichroism can be represented by the matrix

$$
\boldsymbol{U}(A, B, C, D)=\left[\begin{array}{cc}
A+i B & -C+i D \\
C+i D & A-i B
\end{array}\right]
$$

where $A, B, C, D$ are generally complex numbers. 
The output intensity which is observed by the detector in the scheme shown in Fig. 1 can be expressed as

$$
I_{\text {out }}=I_{0} \vec{R}^{T}\left(\phi_{1}, \phi_{2}\right) \bar{M}(A, B, C, D) \vec{S}\left(\phi_{3}, \phi_{4}\right),
$$

where $I_{0}$ is the overall intensity of the probe beam and the vectors $\vec{R}, \vec{S}$, and matrix $\bar{M}$ are given by

$$
\begin{aligned}
& \vec{R}\left(\phi_{1}, \phi_{2}\right)=\left[\begin{array}{c}
1 \\
\frac{1}{2}\left\{\cos 4 \phi_{1}+\cos \left[4\left(\phi_{1}-\phi_{2}\right)\right]\right\} \\
\frac{1}{2}\left\{\sin 4 \phi_{1}-\sin \left[4\left(\phi_{1}-\phi_{2}\right)\right]\right\} \\
-\sin \left[2\left(2 \phi_{1}-\phi_{2}\right)\right]
\end{array}\right], \\
& \vec{S}\left(\phi_{3}, \phi_{4}\right)=\left[\begin{array}{c}
\frac{1}{2}\left\{\cos 2 \phi_{4}+\cos \left[2\left(\phi_{4}-2 \phi_{3}\right)\right]\right\} \\
\frac{1}{2}\left\{\sin 2 \phi_{4}-\sin \left[2\left(\phi_{4}-2 \phi_{3}\right)\right]\right\} \\
\sin \left[2\left(\phi_{4}-\phi_{3}\right)\right]
\end{array}\right],
\end{aligned}
$$

and $\bar{M}$ is $4 \times 4$ real matrix which is the bearer of the information about the optical system under investigation with the components

$$
\begin{aligned}
& M_{11}=\frac{1}{2}\left[|A|^{2}+|B|^{2}+|C|^{2}+|D|^{2}\right], \\
& M_{12}=-\operatorname{Im}\left\{A B^{*}-C D^{*}\right\}, \\
& M_{13}=-\operatorname{Im}\left\{A D^{*}-B C^{*}\right\}, \\
& M_{14}=\operatorname{Im}\left\{A C^{*}+B D^{*}\right\}, \\
& M_{21}=\operatorname{Im}\left\{A B^{*}+C D^{*}\right\}, \\
& M_{22}=\frac{1}{2}\left[|A|^{2}+|B|^{2}-|C|^{2}-|D|^{2}\right], \\
& M_{23}=-\operatorname{Re}\left\{A C^{*}+B D^{*}\right\}, \\
& M_{24}=-\operatorname{Re}\left\{A D^{*}-B C^{*}\right\}, \\
& M_{31}=\operatorname{Im}\left\{A D^{*}+B C^{*}\right\}, \\
& M_{32}=\operatorname{Re}\left\{A C^{*}-B D^{*}\right\}, \\
& M_{33}=\frac{1}{2}\left[|A|^{2}-|B|^{2}-|C|^{2}+|D|^{2}\right], \\
& M_{34}=\operatorname{Re}\left\{A B^{*}+C D^{*}\right\}, \\
& M_{41}=-\operatorname{Im}\left\{A C^{*}-B D^{*}\right\}, \\
& M_{42}=\operatorname{Re}\left\{A D^{*}+B C^{*}\right\}, \\
& M_{43}=-\operatorname{Re}\left\{A B^{*}-C D^{*}\right\}, \\
& M_{44}=\frac{1}{2}\left[|A|^{2}-|B|^{2}+|C|^{2}-|D|^{2}\right] .
\end{aligned}
$$

Taking into account the form of the $\bar{M}$ matrix components (6), several conclusions can be drawn. For example, it is clear that the output intensity $I_{\text {out }}$ is the linear combination of the elements of $\bar{M}$-matrix and the coefficients of this linear combination are generally the functions of the angles $\phi_{1}-\phi_{4}$. The general number of non-zero $\bar{M}$-matrix components is 16 and $M_{11}$ is identically equal to unity for non-depolarizing system. The systems which turn a part of the laser beam into non-polarized light are not taken into account in this paper. The number of non-zero M-components is further reduced when some physical phenomena are not present in the sample, $\bar{M}$-matrix of purely birefringent sample has 10 non-zero components, while $\bar{M}$ matrix of purely dichroic sample has 16 non-zero compenets from which there are 10 independent. Both vectors $\vec{R}$ and $\vec{S}$ can be reduced to just 2 non-zero components by the proper choice of the rotation angles of the wave-plates (angles $\phi_{1}-\phi_{4}$ ) according to Tab. 2 .

\begin{tabular}{ll|lll||ll|lll}
$\phi_{1}$ & $\phi_{2}$ & $\mathrm{R}_{2}$ & $\mathrm{R}_{3}$ & $\mathrm{R}_{4}$ & $\phi_{3}$ & $\phi_{4}$ & $\mathrm{~S}_{2}$ & $\mathrm{~S}_{3}$ & $\mathrm{~S}_{4}$ \\
\hline 0 & 0 & 1 & 0 & 0 & 0 & $3 \pi / 4$ & 0 & 0 & -1 \\
0 & $\pi / 4$ & 0 & 0 & 1 & 0 & $\pi / 4$ & 0 & 0 & 1 \\
$\pi / 8$ & $\pi / 4$ & 0 & 1 & 0 & 0 & 0 & 1 & 0 & 0 \\
$\pi / 4$ & 0 & -1 & 0 & 0 & $\pi / 4$ & $\pi / 4$ & 0 & 1 & 0 \\
- & - & - & - & - & $\pi / 2$ & $\pi / 2$ & -1 & 0 & 0 \\
- & - & - & - & - & $3 \pi / 4$ & $3 \pi / 4$ & 0 & -1 & 0
\end{tabular}

Table 2. Angles $\phi_{1}-\phi_{4}$ reducing the number of non-zero components of $\vec{R}$ and $\vec{S}$

By using values of $\mathrm{R}$ and $\mathrm{S}$ components collected in Tab. 2, the vectors $\vec{R}$ and $\vec{S}$ can be substituted by the matrices $\bar{R}$ and $\hat{S}$ defined as

$$
\begin{aligned}
\bar{R} & =\left[\begin{array}{cccc}
1 & 1 & 0 & 0 \\
1 & 0 & 0 & 1 \\
1 & 0 & 1 & 0 \\
1 & -1 & 0 & 0
\end{array}\right], \\
\hat{S}^{T} & =\left[\begin{array}{cccc}
1 & 0 & 0 & -1 \\
1 & 0 & 0 & 1 \\
1 & 1 & 0 & 0 \\
1 & 0 & 1 & 0 \\
1 & -1 & 0 & 0 \\
1 & 0 & -1 & 0
\end{array}\right] .
\end{aligned}
$$

\begin{tabular}{|c|c|c|c|c|c|c|}
\hline$l_{i \mu}$ & $\begin{array}{c}\text { HWP } \\
\phi_{1} \\
\text { [rad] }\end{array}$ & $\begin{array}{c}\text { QWP } 1 \\
\phi_{2} \\
{[\mathrm{rad}]}\end{array}$ & $\begin{array}{c}\text { QWP } 2 \\
\phi_{3} \\
\text { [rad] }\end{array}$ & $\begin{array}{c}\text { P } 2 \\
\phi_{4} \\
\text { [rad] }\end{array}$ & $\begin{array}{c}I_{\text {out }} \\
\left(\phi_{1}, \phi_{2}, \phi_{3}, \phi_{4}\right)\end{array}$ & $\begin{array}{l}\text { Probe } \\
\text { Pol. }\end{array}$ \\
\hline$\overline{I_{11}}$ & 0 & 0 & 0 & $3 \pi / 4$ & 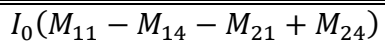 & $\overline{\bar{\top} \uparrow}$ \\
\hline $\mathrm{I}_{12}$ & 0 & 0 & 0 & $\pi / 4$ & $I_{0}\left(M_{11}+M_{14}-M_{21}-M_{24}\right)$ & $\uparrow$ \\
\hline $\mathrm{I}_{13}$ & 0 & 0 & 0 & 0 & $I_{0}\left(M_{11}+M_{12}-M_{21}+M_{22}\right)$ & $\uparrow$ \\
\hline $\mathrm{I}_{14}$ & 0 & 0 & $\pi / 4$ & $\pi / 4$ & $I_{0}\left(M_{11}+M_{13}-M_{21}-M_{23}\right)$ & $\uparrow$ \\
\hline $\mathrm{I}_{15}$ & 0 & 0 & $\pi / 2$ & $\pi / 2$ & $I_{0}\left(M_{11}-M_{12}-M_{21}-M_{22}\right)$ & $\uparrow$ \\
\hline$I_{16}$ & 0 & 0 & $3 \pi / 4$ & $3 \pi / 4$ & $I_{0}\left(M_{11}-M_{13}-M_{21}+M_{23}\right)$ & $\uparrow$ \\
\hline$I_{21}$ & 0 & $\pi / 4$ & 0 & $3 \pi / 4$ & $I_{0}\left(M_{11}-M_{14}-M_{41}-M_{44}\right)$ & $\bar{v}$ \\
\hline $\mathrm{I}_{22}$ & 0 & $\pi / 4$ & 0 & $\pi / 4$ & $I_{0}\left(M_{11}+M_{14}-M_{41}+M_{44}\right)$ & $v$ \\
\hline $\mathrm{I}_{23}$ & 0 & $\pi / 4$ & 0 & 0 & $I_{0}\left(M_{11}+M_{12}-M_{41}-M_{42}\right)$ & $v$ \\
\hline $\mathrm{I}_{24}$ & 0 & $\pi / 4$ & $\pi / 4$ & $\pi / 4$ & $I_{0}\left(M_{11}+M_{13}-M_{41}-M_{43}\right)$ & $\circlearrowleft$ \\
\hline $\mathrm{I}_{25}$ & 0 & $\pi / 4$ & $\pi / 2$ & $\pi / 2$ & $I_{0}\left(M_{11}-M_{12}-M_{41}+M_{42}\right)$ & $\checkmark$ \\
\hline $\mathrm{I}_{26}$ & 0 & $\pi / 4$ & $3 \pi / 4$ & $3 \pi / 4$ & $I_{0}\left(M_{11}-M_{13}-M_{41}+M_{43}\right)$ & 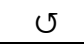 \\
\hline$I_{31}$ & $\pi / 8$ & $\pi / 4$ & 0 & $3 \pi / 4$ & $I_{0}\left(M_{11}-M_{14}-M_{31}+M_{34}\right)$ & $\pi$ \\
\hline $\mathrm{I}_{32}$ & $\pi / 8$ & $\pi / 4$ & 0 & $\pi / 4$ & $I_{0}\left(M_{11}+M_{14}-M_{31}-M_{34}\right)$ & $\lambda$ \\
\hline
\end{tabular}

Using these new matrices, the intensity matrix

$$
\hat{I}=I_{0} \bar{R} \bar{M} \hat{S},
$$

can be calculated. The components of the matrix $\hat{I}$ are directly measurable and can be used for the complete reconstruction of the Jones matrix of the unknown optical component or set of optical components characterized by the general Jones matrix $\boldsymbol{U}$ given by (2). It should be noted that the components of the matrices $\bar{R}, \bar{M}$, and $\hat{S}$ are all real. The Jones matrix $\boldsymbol{U}$ can be reconstructed from the intensity matrix $\hat{I}$ up to the multiplicative complex constant, which however does not affect the polarization state of the beam and contains just the information about the absolute phase of the laser beam. The measured components of the intensity matrix $\hat{I}$ with wave-plates angles, connection between measured intensity and elements of $\bar{M}$ matrix and the probe beam polarization state are summarized in Tab. 3. 


\begin{tabular}{l||c|c|c|c||c|c}
$\mathrm{I}_{33}$ & $\pi / 8$ & $\pi / 4$ & 0 & 0 & $I_{0}\left(M_{11}+M_{12}-M_{31}-M_{32}\right)$ & $\nearrow$ \\
$\mathrm{I}_{34}$ & $\pi / 8$ & $\pi / 4$ & $\pi / 4$ & $\pi / 4$ & $I_{0}\left(M_{11}+M_{13}-M_{31}+M_{33}\right)$ & $\nearrow$ \\
$\mathrm{I}_{35}$ & $\pi / 8$ & $\pi / 4$ & $\pi / 2$ & $\pi / 2$ & $I_{0}\left(M_{11}-M_{12}-M_{31}+M_{32}\right)$ & $\nearrow$ \\
$\mathrm{I}_{36}$ & $\pi / 8$ & $\pi / 4$ & $3 \pi / 4$ & $3 \pi / 4$ & $I_{0}\left(M_{11}-M_{13}-M_{31}-M_{33}\right)$ & $\nearrow$ \\
\hline $\mathrm{I}_{41}$ & $\pi / 4$ & 0 & 0 & $3 \pi / 4$ & $I_{0}\left(M_{11}-M_{14}+M_{21}-M_{24}\right)$ & $\rightarrow$ \\
$\mathrm{I}_{42}$ & $\pi / 4$ & 0 & 0 & $\pi / 4$ & $I_{0}\left(M_{11}+M_{14}+M_{21}+M_{24}\right)$ & $\rightarrow$ \\
$\mathrm{I}_{43}$ & $\pi / 4$ & 0 & 0 & 0 & $I_{0}\left(M_{11}+M_{12}+M_{21}-M_{22}\right)$ & $\rightarrow$ \\
$\mathrm{I}_{44}$ & $\pi / 4$ & 0 & $\pi / 4$ & $\pi / 4$ & $I_{0}\left(M_{11}+M_{13}+M_{21}+M_{23}\right)$ & $\rightarrow$ \\
$\mathrm{I}_{45}$ & $\pi / 4$ & 0 & $\pi / 2$ & $\pi / 2$ & $I_{0}\left(M_{11}-M_{12}+M_{21}+M_{22}\right)$ & $\rightarrow$ \\
$\mathrm{I}_{46}$ & $\pi / 4$ & 0 & $3 \pi / 4$ & $3 \pi / 4$ & $I_{0}\left(M_{11}-M_{13}+M_{21}-M_{23}\right)$ & $\rightarrow$ \\
\hline
\end{tabular}

Table 3. List of experimetally measured intensities, corresponding wave-plates angles, linear combinations of $\bar{M}$ matrix components and probe beam polarization state $: \uparrow, \nearrow, \rightarrow$ - linear polarization directed along the arrow, $\cup$ - left-handed circular polarization

\subsection{Experimental determination of $M$ matrix}

In order to determine the complete Jones matrix of the studied sample, it is needed to measure the intensity matrix $\hat{\boldsymbol{I}}$ by the procedure summarized in the Tab. 3 . The first step of the Jones matrix reconstruction would be the elimination of the stray and background light by the subtraction of the specific pairs of intensities. Let us denote the matrix of background light free intensities by $\bar{J}$, where

$$
\bar{J}=\bar{J}^{\prime}+\bar{J}^{\prime \prime}
$$

Here,

$$
J_{i j}^{\prime}=I_{i \mu} V^{\prime}{ }_{\mu j}
$$

and

$$
J^{\prime \prime}{ }_{i j}=\left\{\begin{array}{l}
\left\|J^{\prime}{ }_{i j}\right\|_{j}, \quad j=1 \\
0, \quad \text { otherwise }
\end{array},\right.
$$

where $\left\|J^{\prime}{ }_{i j}\right\|_{j}$ is a 2-norm of $\mathrm{j}$-th row of $\bar{J}^{\prime}$ matrix and

$$
\widehat{V}^{\prime}=\left[\begin{array}{cccc}
0 & 0 & 0 & -1 \\
0 & 0 & 0 & 1 \\
0 & 1 & 0 & 0 \\
0 & 0 & 1 & 0 \\
0 & -1 & 0 & 0 \\
0 & 0 & -1 & 0
\end{array}\right] .
$$

The elements of $\bar{M}$-matrix are given as

$$
M_{i j}=\frac{1}{4 I_{0}} W_{i k} J_{k j}
$$

where

$$
\bar{W}=\left[\begin{array}{cccc}
1 & 0 & 0 & 1 \\
1 & 0 & 0 & -1 \\
-1 & 0 & 2 & -1 \\
-1 & 2 & 0 & -1
\end{array}\right]
$$

The $\bar{M}$-matrix can be used directly for the reconstruction of the Jones matrix of the optical assembly under investigation.

\subsection{Reconstruction of the Jones matrix}

Let us define two-fold of the skew-symmetric part of the $\bar{M}$ matrix as

$$
\bar{N} \equiv \bar{M}-\bar{M}^{T} .
$$

Using this definition, the Jones matrix can be obtained in the form

where

$$
\boldsymbol{U}=\frac{A}{\operatorname{Tr}\{\bar{M}\}}\left[\begin{array}{ll}
U_{11} & U_{12} \\
U_{21} & U_{22}
\end{array}\right]
$$

$$
\begin{gathered}
U_{11}=\operatorname{Tr}\{\bar{M}\}-N_{12}+i N_{34}, \\
U_{12}=-N_{13}+N_{23}+i\left(N_{14}-N_{24}\right), \\
U_{21}=-N_{13}-N_{23}-i\left(N_{14}+N_{24}\right), \\
U_{22}=\operatorname{Tr}\{\bar{M}\}+N_{12}-i N_{34} .
\end{gathered}
$$

Here, $\operatorname{Tr}\{\bar{M}\}$ stands for the trace of the matrix. It should be noted that the factor $1 / 410$ from (13) is unsubstantial, while it does not influence the polarization state of the light and therefore it has been dropped. The multiplicative complex factor $A$ influences just the total isotropic phase and absorption, but cannot change the polarization state of the light and as such it can be also considered to be equal to unity.

2.4 Determination of the polarization influencing phenomena With the knowledge of the Jones matrix of the optical system one can use the procedure derived by Jones [Jones 1948] to determine the contributions of physical phenomena contributing to the change of the polarization state of the passing through light like linear and circular birefringence which is the phase delay between two eigenpolarizations as well as linear and circular dichroism describing the different absorption of eigenpolarizations.

For this purpose, it is convenient to calculate the differential Jones matrix $\mathrm{N}$ respective to the obtained Jones matrix $\mathrm{U}$.

$$
\boldsymbol{N}=\left[\begin{array}{cc}
F_{U}+\frac{1}{2}\left(U_{11}-U_{22}\right) G_{U} & U_{12} G_{U} \\
U_{21} G_{U} & F_{U}-\frac{1}{2}\left(U_{11}-U_{22}\right) G_{U}
\end{array}\right],
$$

where

$$
\begin{gathered}
F_{U}=\frac{1}{L} \ln \left[ \pm_{1} \sqrt{\operatorname{det}\{\boldsymbol{U}\}}\right], \\
G_{U}=\frac{1}{L Q_{U}} \ln \left[\frac{2 Q_{U} \pm_{2} \operatorname{Tr}\{\boldsymbol{U}\}}{2 \exp \left[L F_{U}\right]}\right],
\end{gathered}
$$

where $L$ is the length of the sample. The discriminant $Q U$ is given by

$$
Q_{U}= \pm \sqrt{\frac{1}{4}\left(M_{11}-M_{22}\right)^{2}+M_{12} M_{21}}
$$

It can be seen directly from (19) and (20) that the reconstruction of $\mathrm{N}$ matrix from $\bar{M}$ is not unique. There are up to eight different $\mathrm{N}$ matrices corresponding the same $\bar{M}$-matrix. These eight matrices are given by the combinations of $\pm_{1}, \pm_{2}$, and $\pm_{3}$. This physically represents the possibility to find different combinations of optical elements leading to the same total influence to the polarization state of the passing through light.

The final extraction of the particular physical phenomena from $\mathrm{N}$ matrix components is summarized in the Tab. 4.

\begin{tabular}{l|c|l}
\hline phenomenon & symbol & Formula \\
\hline Linear birefringence 0 & $g_{0}$ & $\frac{1}{2} \operatorname{Im}\left\{N_{11}-N_{22}\right\}$ \\
Linear dichroism 0 & $p_{0}$ & $\frac{1}{2} \operatorname{Re}\left\{N_{11}-N_{22}\right\}$ \\
Linear birefringence 45 & $g_{45}$ & $\frac{1}{2} \operatorname{Im}\left\{N_{21}+N_{12}\right\}$ \\
Linear dichroism 45 & $p_{45}$ & $\frac{1}{2} \operatorname{Re}\left\{N_{21}+N_{12}\right\}$ \\
Circular birefringence & $\omega$ & $\frac{1}{2} \operatorname{Re}\left\{N_{21}-N_{12}\right\}$
\end{tabular}


Circular dichroism

$$
\begin{array}{l|l}
\delta & \frac{1}{2} \operatorname{Im}\left\{N_{21}-N_{12}\right\}
\end{array}
$$

Table 4. Extraction of physical phenomena from $\mathrm{N}$ matrix

For example, linear birefringence 45 means the linear birefringence with the birefringence axis directed under the angle of 45 degrees with respect to the $x$-axis.

The linear birefringence and dichroism is very often represented by the total birefringence $g$ and total dichroism $p$ together with birefringence axes azimuth $\theta_{g}$ and dichroism axes azimuth $\theta_{p}$ given by

$$
\begin{array}{ll}
g=\sqrt{g_{0}^{2}+g_{45}^{2}}, & \theta_{g}=\frac{1}{2} \tan ^{-1} \frac{g_{45}}{g_{0}}, \\
p=\sqrt{p_{0}^{2}+p_{45}^{2}}, & \theta_{p}=\frac{1}{2} \tan ^{-1} \frac{p_{45}}{p_{0}} .
\end{array}
$$

It should be noted that the whole measurement procedure can be done as a spatially resolved measurement using a camera as a detector in order to observe also spatially non-homogenous phenomena like birefringence or dichroism induced by the mechanical stress or some external force field.

\section{SIMULATED MEASUREMENT AND RESULTS ANALYSIS}

Let us now simulate the analysis of a unit length sample with inhomogeneous distribution of the linear and circular birefringence and dichroism. Let the sample have a cylindrical shape with the linear birefringence $g$ distributed throughout the sample cross-section according to

$$
g(r)=-\frac{\pi}{4} r^{2}
$$

and the circular birefringence $\omega$ according to

$$
\omega(r)=\frac{\pi}{8} r^{2}+\frac{\pi}{8}
$$

Such birefringence distribution corresponds qualitatively for example to the heat-loaded piece of magneto-active optical material with temperature-dependent Verdet constant (responsible for Faraday rotation) in the axial magnetic field. Let us also include the linear $p$ and circular $\delta$ dichroism as

$$
p(r)=-\frac{\pi}{16} r^{2}
$$

and

$$
\delta(r)=\frac{\pi}{16} r^{2}+\frac{\pi}{16}
$$

According to (21) the birefringence and dichroism indicators $g_{0}$ $\delta$ are shown in Fig. 2.
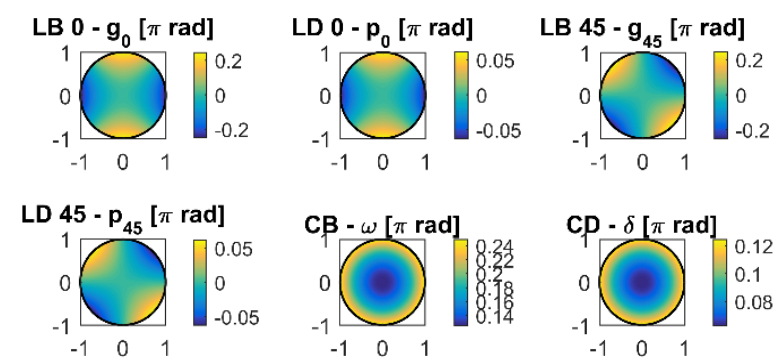

Figure 2. Birefringence and dichroism indicators of the simulated sample.

The measurement error arising from the deviation of the azimuth angle $\phi_{1}-\phi_{4}$ of the wave-plates can be simulated by the addition of the angle deviation $\Delta \phi_{i}$ with the normal distribution. In our case the Gaussian noise with $\sigma=0.6 \mathrm{deg}$ has been used. The deviations of $\phi$ angles for the measurement of each particular intensity is shown in the Fig. 3

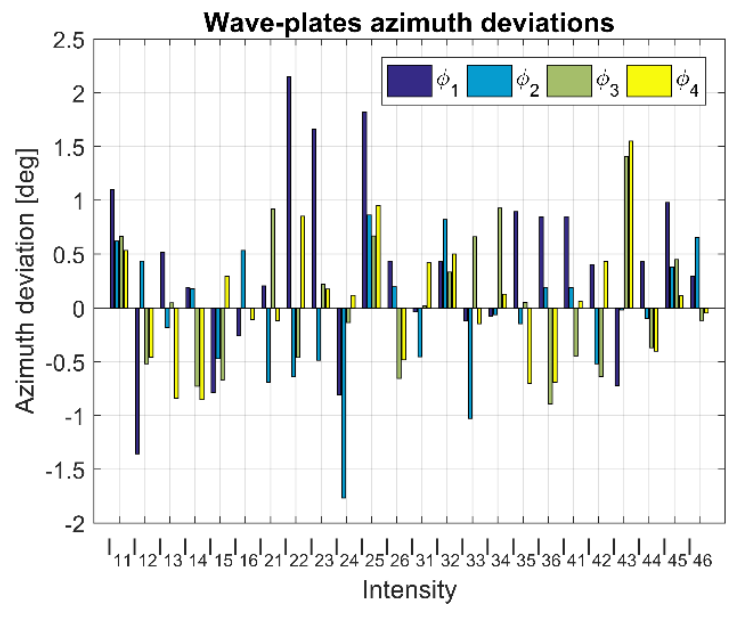

Figure 3. Azimuth angles $\phi_{1}-\phi_{4}$ deviations

Using the above summarized parameters and errors, one is able to generate the $\hat{I}$-matrix according to (8). The resulting intensity profiles arranged into $\hat{I}$-matrix as they could be observed on the detector are shown in Fig. 4

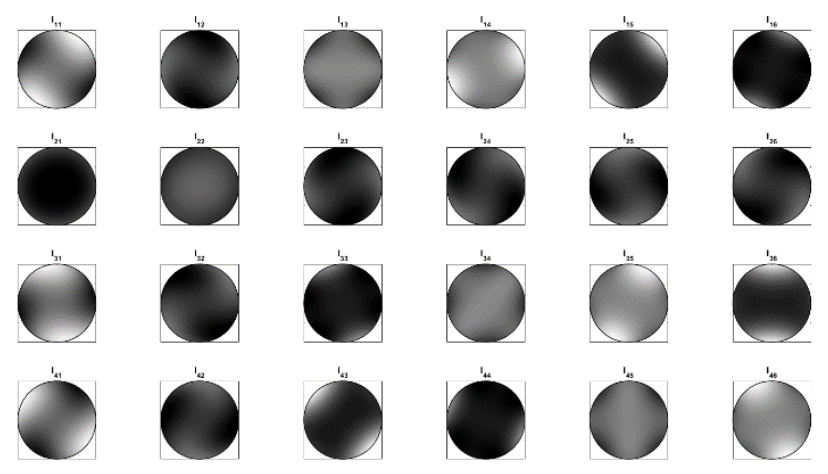

Figure 4. Generated $\hat{I}$-matrix including the simulated errors arisen due to the angular misalignment of the wave-plates.

The $\hat{I}$-matrix array will be the only input into the Jones matrix array reconstruction process.

Using equations (13), (16), and (17) one can reconstruct the Jones matrices array. The resulting contributions of the birefringence and dichroism components can be then obtained from the Jones matrices using equation (18) and Tab. 4. As mentioned above, this reconstruction is not unique. In this particular case four possible realizations have been found. The first one, which corresponds to the input parameters is shown in Fig. 5
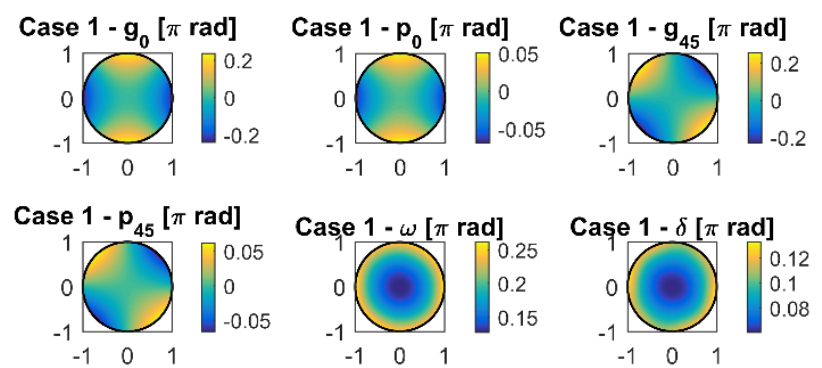

Figure 5. Reconstructed birefringence and dichroism indicators of the simulated sample - case 1 .

The other possible realization is shown in Fig. 6 

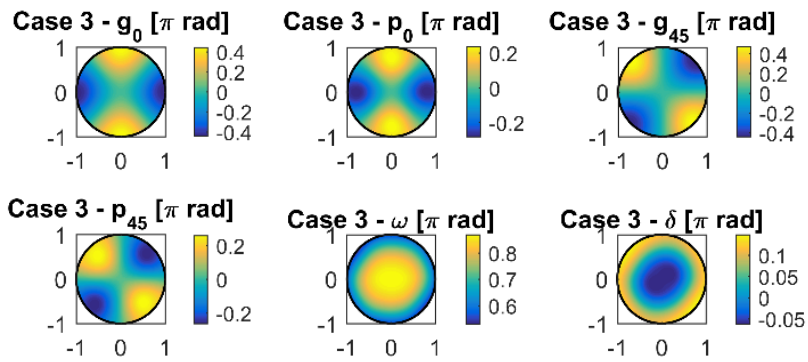

Figure 6. Reconstructed birefringence and dichroism indicators of the simulated sample - case 2 .

The other realizations can be obtained by the multiplication of the preceding ones by -1 . The result which corresponds to the generated sample shown in the Fig. 2 is the case 1 shown in the Fig. 5 . There are just minor deviations caused by the generated adjustment errors shown in the Fig. 3 . It should be noted, that all the reconstructed combinations of birefringence and dichroism components are leading to the same total Jones matrix of the optical system.

\section{CONCLUSIONS}

A polarimetric method suitable for the characterization of full Jones matrix of the optical components or systems has been proposed including the method for the extraction of the particular physical phenomena influencing the polarization of the laser beam like linear and circular birefringence or dichroism. This method allows the spatially resolved measurement suitable for the measurement of inhomogeneously distributed birefringence or dichroism. The knowledge of the complete Jones matrix also allows the subtraction of a part of more complex optical system in order to obtain the Jones matrix of the rest of the system.

This method has been demonstrated for the analysis of the simulated sample containing spatially inhomogenous distribution of both linear and circular birefringence and dichroism. It has been shown, that it is possible to reconstruct the Jones matrices array corresponding to such sample and also to extract the particular birefringences and dichroisms.

This method could be very useful for the analysis of the induced polarization effects generated in complex high power laser systems. Experiments will be conducted to confirm the validity of this method.

\section{ACKNOWLEDGMENTS}

This work was co-financed by the European Regional Development Fund and the state budget of the Czech Republic (project HiLASE CoE: Grant No. CZ.02.1.01/0.0/0.0/15_006/0000674) and by the European Union's Horizon 2020 research and innovation programme under grant agreement No. 739573. This work was also supported by the Ministry of Education, Youth and Sports of the Czech Republic (Programmes NPU I Project No. LO1602, and Large Research Infrastructure Project No. LM2015086).

\section{REFERENCES}

[Mason 2017] Mason, P., et al. Kilowatt average power $100 \mathrm{~J}$ level diode pumped solid state laser. Optica, April 2017, Vol. 4, No. 4, pp 438-439, ISSN 2334-2536
[Reagan 2018] Reagan, B.A., et al. Scaling diode-pumped, high energy picosecond lasers to kilowatt average powers. HPLSE, 2018, Vol. 6, No. e11, pp 1-9, ISSN 2052-3289

[Zapata 2015] Zapata, L.E., et al. Cryogenic Yb:YAG compositethin-disk for high energy and average power amplifiers. Opt. Lett., May 2015, Vol. 40, No. 11, pp 2610-2613, ISSN 1539-4794

[Novak 2015] Novak, O., et al. Status of the high average power diode-pumped solid state laser development at HiLASE. Appl. Sci., 2015, Vol. 5, No. 4, pp 637-665, ISSN 2076-3417

[Koechner 1970] Koechner, W., et al. Thermal Lensing in a Nd:YAG Laser Rod. Appl. Opt., November 1970, Vol. 9, No. 11, pp 2548-2553, ISSN 2155-3165

[Slezak 2013] Slezak, O., et al. Optimization of Wavefront Distortions and Thermal-Stress Induced Birefringence in a Cryogenically-Cooled Multislab Laser Amplifier. IEEE J. Quantum Electron., September 2013, Vol. 49, No. 11, pp 960-966, ISSN 1558-1713

[Graupeter 2014] Graupeter, T., et al. Calculations of Eigenpolarization in Nd:YAG Laser Rods Due to Thermally Induced Birefringence. IEEE J. Quantum Electron., October 2014, Vol. 50, No. 12, pp 10351043 , ISSN 1558-1713

[Slezak 2015] Slezak, O., et al. Thermally induced depolarization in terbium gallium garnet ceramics rod with natural convection cooling. J. Opt., May 2015, Vol. 17, No. 6, pp 065610, ISSN 2040-8986

[Ribes-Pleguezuelo 2017] Ribes-Pleguezuelo, P., et al. Method to simulate and analyse induced stresses for laser crystal packaging technologies. Opt. Ex., March 2017, Vol. 25, No. 6, pp 5927-5940, ISSN 1094-4087

[Körner 2017] Körner, J., et al. Spatially and temporally resolved temperature measurement in laser media. Opt. Lett., May 2016, Vol. 41, No. 11, pp 2525-2528, ISSN 15394794

[Chi 2018] Chi, H., et al. In situ 3-D temperature mapping of high average power cryogenic laser amplifiers. Opt. Ex., February 2018, Vol. 26, No. 5, pp 5240-5252, ISSN 1094-4087

[Lumer 2010] Lumer, Y., et al. Depolarization induced by pump edge effects in high average power laser rods. JOSA B, January 2010, Vol. 27, No. 1, pp 38-44, ISSN 15208540

[Yasuhara 2013] Yasuhara, R. and Furuse, H. Thermally induced depolarization in TGG ceramics. Opt. Lett., May 2013, Vol. 38, No. 10, pp 1751-1753, ISSN 1539-4794

[Kagan 2003] Kagan, M.A. and Khazanov, E.A. Compensation for thermally induced birefringence in polycrystalline ceramic active elements. Quantum Electron., 
January 2003, Vol. 33 , No. 10 , pp $876-882$, ISSN 1468-4799

[Snetkov 2011] Snetkov, I., et al. Compensation of thermally induced depolarization in Faraday isolators for high average power lasers. Opt. Ex., March 2011, Vol. 19, No. 7, pp 6366-6376, ISSN 1094-4087

[Goldstein 1992] Goldstein, D. H. Mueller matrix dual-rotating retarder polarimeter. Appl. Opt., November 1992, Vol. 31, No. 31 pp 6676-6683, ISSN 2155-3165

[Savenkov 2007] Savenkov, S. N., Sydoruk, O. I., and Muttiah, R. $\mathrm{S}$. Eigenanalysis of dichroic, birefringent, and degenerate

\section{CONTACTS:}

Ing. Ondřej Slezák, Ph.D.

Dr. Antonio Lucianetti

HiLASE Centre, Institute of Physics, Czech Academy of Sciences, Za Radnicí 828, Dolní Břežany, 252 41, Czech Republic

(+420) 3140077 32, ondrej.slezak@hilase.cz polarization elements: a Jones-calculus study. Appl. Opt., September 2007, Vol. 46, No. 27, pp 6700-6709, ISSN 2155-3165

[Azzam 2016] Azzam, R.M.A. Stokes-vector and Mueller-matrix polarimetry. JOSA A, July 2016, Vol. 33, No. 7, pp 1396-1408, ISSN 1520-8532

[Jones 1948] Jones, J.C. A New Calculus for the Treatment of Optical Systems. VII. Properties of the N-matrices. JOSA, August 1948, Vol. 38, No. 8, pp 671-685, ISSN 0030-3941 